Check for updates

Cite this: RSC Adv., 2019, 9, 2591

Received 18th November 2018 Accepted 10th January 2019

DOI: $10.1039 / c 8 r a 09482 a$

rsc.li/rsc-advances

\section{Modulation of energy metabolism and mitochondrial biogenesis by a novel proteoglycan from Ganoderma lucidum}

\author{
Zhou Yang, ${ }^{a}$ Zeng Zhang, ${ }^{b}$ Juan Zhao, ${ }^{a}$ Yanming He, ${ }^{b}$ Hongjie Yang ${ }^{* b}$ \\ and Ping Zhou (D)*a
}

\begin{abstract}
In this study we first focused on the effects of a novel proteoglycan extracted from Ganoderma lucidum (FYGL) on mitochondrial biogenesis, because mitochondrial dysfunction is highly related to insulin resistance. We found that FYGL can decrease ROS levels and increase ATP content in rat skeletal muscle L6 cells. In PGC$1 \alpha$ silent cells, FYGL increased expression of PGC-1 $\alpha$ and positively modulated the Sirt1/PGC-1 $\alpha$ pathway. Moreover, FYGL orally administered up-regulated the mitochondrial DNA (mtDNA) copy number and the related gene expressions that control mitochondrial biogenesis in ob/ob mice. Our work well elucidated the mechanism of FYGL ameliorating insulin resistance in the aspect of energy metabolism.
\end{abstract}

\section{Introduction}

The mitochondrion is an important intracellular organelle and mediates a series of biochemical reactions from energy production to apoptosis. ${ }^{1}$ Mitochondria control the oxidative respiratory chain and produce reactive oxygen species (ROS), whereas excessive ROS will impair mitochondrial DNA (mtDNA) and cause intracellular reaction dysfunction. ${ }^{1}$ It is notable that mitochondria play an important role in controlling type 2 diabetes mellitus (T2DM) by regulating the energy balance. It has been a hot topic recently to reveal the relationship between mitochondrial function and insulin resistance. ${ }^{2,3}$ Insulin resistance in T2DM usually accompanies mitochondrial dysfunction including reduction of oxidative phosphorylation activity. ${ }^{4}$ In turn, mitochondrial dysfunction also promotes insulin resistance and produces more ROS. ${ }^{4}$ ROS induces inactivation of insulin signaling pathway, and is considered as one of the major factors in the development of T2DM. ${ }^{5}$ In addition, mitochondrial density and volume are decreased in T2DM patients, which are associated with down-regulation of the peroxisome proliferator-activated receptor $\gamma$ coactivator- $1 \alpha$ (PGC-1 $\alpha$ ) expression and the adenosine-triphosphate (ATP) content. $^{6-9}$

Mitochondrial biogenesis is highly correlated with PGC-1 $\alpha$, which is praised as the master regulator of mitochondrial biogenesis and energy expenditure. ${ }^{\mathbf{1 0}}$ Dysfunction in

${ }^{a}$ State Key Laboratory of Molecular Engineering of Polymers, Department of Macromolecular Science, Fudan University, Shanghai 200433, P. R. China. E-mail: pingzhou@fudan.edu.cn; Fax: (+86)-21-31244038; Tel: (+86)-21-31244038

${ }^{5}$ Yueyang Hospital of Integrated Traditional Chinese and Western Medicine, Shanghai University of Traditional Chinese Medicine, Shanghai 200437, P. R. China. E-mail: yanghongjie1964@aliyun.com mitochondrial energy metabolism decreases ATP production and generates more ROS, while intracellular PGC- $1 \alpha$ can detoxify ROS via mediating ROS detoxifying enzymes. ${ }^{11}$ As one of the mammalian homology of yeast silent information regulator 2 (Sir2), sirtuin 1 (Sirt1) can mediate energy metabolism, and has been an attractive drug target in recent years. ${ }^{10}$ Sirt1 is an upstream effector of PGC- $1 \alpha$ and activates PGC- $1 \alpha$ through deacetylation. It is reported that activation of Sirt1 will ameliorate insulin resistance in mammalian peripheral tissues, ${ }^{12}$ and overexpression of Sirt1 in skeletal muscle tissues can reduce ROS and improve insulin sensitivity. ${ }^{13}$

Our previous work reported that a novel protein tyrosine phosphate 1B (PTP1B) inhibitor extracted from Ganoderma lucidum, named FYGL, can regulate insulin signaling pathway and promote the activation of adenosine $5^{\prime}$-monophosphate activated protein kinase (AMPK) in skeletal muscle tissues of ob/ob mice. ${ }^{14,15}$ Therefore, we first focused on mitochondrial biogenesis regulated by FYGL in the present study. We found that FYGL recovered the mtDNA copy number to normal level in skeletal muscle tissues of ob/ob mice, and positively mediated Sirt1/PGC-1 $\alpha$ pathway and ameliorated mitochondrial dysfunction caused by insulin resistance via decreasing ROS level, and increasing ATP production in L6 cells. Our work revealed that FYGL ameliorated insulin resistance through positive modulation of mitochondrial biogenesis from the point of energy metabolism in vivo.

\section{Materials and methods}

\subsection{Materials}

FYGL was extracted from Ganoderma lucidum in our laboratory. ${ }^{\mathbf{1 4}}$ Dulbecco's modified Eagle's medium (DMEM), fetal bovine serum (FBS) were purchased from Gibco Co. Ltd. (USA). 2',7'- 
Dichlorodihydrofluorescein diacetate (DCFH-DA) and genomic DNA kit were purchased from Sigma Co. Ltd. (Shanghai, China). BCA (bicinchoninic acid) kit, PBS, penicillin and streptomycin were acquired from Sangon Co. Ltd. (Shanghai, China). 2.5\% glutaraldehyde, trizol and SYBR Green I were provided by Yeasen Co. Ltd. (Shanghai, China). RIPA lysis buffer and ATP assay kit were bought from Beyotime Co. Ltd. (Shanghai, China). L6 cells were provided by JRDUN Co. Ltd. (Shanghai, China).

\subsection{Animal trial}

All animal procedures were performed in accordance with the Guidelines for Care and Use of Laboratory Animals of Fudan University and approved by the Animal Ethics Committee of Medical College. 10 male eight-week-old wild type (WT) C57BL/ $6 \mathrm{~J}$ mice and 50 male eight-week-old obese C57BL/6J (ob/ob) mice were obtained from Chinese Academy of Sciences Shanghai Institute of Materia Medica. All mice were housed at $22 \pm 2{ }^{\circ} \mathrm{C}$ and $45-75 \%$ relative humidity with a $12 \mathrm{~h}$ light/dark cycle. $50 \mathrm{ob} / \mathrm{ob}$ mice were randomly divided into 5 groups with 10 mice in each group, model, given FYGL $(150,300,400 \mathrm{mg}$ $\mathrm{kg}^{-1}$ ) and metformin ( $250 \mathrm{mg} \mathrm{kg}^{-1}$ ) once a day, respectively. All mice were killed and the skeletal muscle tissues were isolated after 4 weeks.

\section{3 mtDNA content assay}

Rat skeletal muscle L6 cell lines were cultured in DMEM with $10 \%$ FBS and $1 \%$ penicillin-streptomycin at $37{ }^{\circ} \mathrm{C}$ in $5 \% \mathrm{CO}_{2}$ atmosphere. Genomic DNA was extracted from skeletal muscle tissues and L6 cells using genomic DNA kit. mtDNA was proliferated by quantitative PCR with the primer (the sequence was shown in Table 1) and the copy number was calculated.

\subsection{RNA extraction and quantitative PCR (qPCR)}

RNA was extracted from skeletal muscle tissues and L6 cells by using trizol agent, and then reversely transcribed to complementary DNA (cDNA). SYBR Green I including Taq enzyme and series of primers $\beta$-actin (as internal reference), Sirt1, sirtuin 3 (Sirt3), PGC-1 $\alpha$, mitochondrial transcription factor A (TFAM) and nuclear respiratory factor-1 (NRF1) (all synthesized by Sangon Co, and their sequences are shown in Table 1) were mixed with cDNA, proliferated on qPCR instrument (Bio-rad, Germany). The relative expression mRNA was determined according to the method in the ref. 16.

\subsection{Protein extraction and western blot}

Skeletal muscle tissues and L6 cells were lysed in RIPA lysis buffer and centrifuged $\left(12000 \times g, 10 \mathrm{~min}, 4^{\circ} \mathrm{C}\right)$. The lysates were separated by $10 \%$ SDS-PAGE and transferred to polyvinylidene fluoride membrane, immunoblotted with following primary antibodies of rabbit monoclonal anti-Sirt1, anti-Sirt3, anti-PGC-1 $\alpha$ (BBI, Shanghai, China), anti-TFAM, anti-NRF1 and anti-GAPDH (ABCAM, Cambridge, UK), and then incubated with secondary goat anti-rabbit antibody (Cell Signaling, USA). The bands were visualized with enhanced chemiluminescence solution (ECL, Biotanon) and detected by Image Lab camera (Bio-rad, Germany), and then quantified by densitometry scanning with ImageJ software.

\subsection{ROS level assay}

Briefly, L6 cells were incubated with $F Y G L$ for $24 \mathrm{~h}$, then $10 \mu \mathrm{mol}$ $\mathrm{L}^{-1}$ DCFH-DA diluted in DMEM with $2 \%$ FBS $(1: 1000)$ was added. After incubation for $20 \mathrm{~min}$, DCFH-DA was removed and the cells were washed with PBS three times, and then ROS level was analyzed by flow cytometry (Gallios, Beckman coulter, USA).

\subsection{Intracellular ATP content assay}

L6 cells were seeded into 6-well plates, and FYGL at designated concentrations was added and incubated for $24 \mathrm{~h}$. Then the cells were washed with cold PBS three times, and lysed by ATP lysis, and then centrifuged to collect supernatant. The emitted light was assayed by microplate luminometer (Bio-Tek, USA) and ATP content was calibrated by protein concentration finally.

\subsection{Transfection with PGC-1 $\alpha$ siRNA}

L6 cells were seeded into 6-well plates. $20 \mu \mathrm{mol} \mathrm{L}{ }^{-1}$ PGC- $1 \alpha$ siRNA (synthesized by Sangon Bio. Co, the sequence was shown

Table 1 The primer sequences

\begin{tabular}{lll}
\hline Gene & Forward primer $\left(5^{\prime}\right.$ to $\left.3^{\prime}\right)$ & Reverse primer $\left(5^{\prime}\right.$ to $\left.3^{\prime}\right)$ \\
\hline Rat $\beta$-actin & CCCATCTATGAGGGTTACGC & TTTAATGTCACGCACGATTTC \\
Rat PGC-1 $\alpha$ & CGCACAACTCAGCAAGTCCTC & CCTTGCTGGCCTCCAAAGTCTC \\
Rat Sirt1 & CAGCATCTTGCCTGATTTGTAA & TTGGAATTAGTGCTACTGGTCTTAC \\
Rat Sirt3 & TCTACAGCAACCTTCAGCAGTATG & AAGGAAGTAGTGAGCGACATTGG \\
Rat mtDNA & AGCTATTAATGGTTCGTTTGT & AGGAGGCTCCATTTCTCTTGT \\
Rat NRF1 & AGAGACAGCAGACACGGTTG & GCTGCGCCAAACACCTTAAA \\
Rat TFAM & AGCAAATGGCTGAAGTTGGG & TCTAGTAAAGCCCGGAAGGT \\
Mouse $\beta$-actin & GGCTGTATTCCCCTCCATCG & CCAGTTGGTAACAATGCCATGT \\
Mouse mtDNA & TAAATTCGTGCCAGCCACC & GTTGACACGTTTTACGCCGA \\
Mouse Sirt1 $1 \alpha$ & ATCTGACCACAAACGATGACCCT & GAGGCATCTTTGAAGTCTAGTTGTCTA \\
Mouse Sirt3 3 & GCTGACGACTTCGACGACG & TCGGTCAACAGGAGGTTGTCT \\
Mouse TFAM & TACAGGCCCAATGTCACTCA & GGATCCCAGATGCTCTCTCA \\
Mouse NRF1 & ATTCCGAAGTGTTTTCCAGCA & TCTGAAAGTTTGCATCTGGGT
\end{tabular}


Table 2 The PGC-1 $\alpha$ siRNA sequence

Sense $\left(5^{\prime}\right.$ to $\left.3^{\prime}\right)$

Anti-sense ( $5^{\prime}$ to $\left.3^{\prime}\right)$

AAGACGGAUUGCCCUCAUUUGtt CAAAUGAGGGCAAUCCGUCUUtt

in Table 2) and $5 \mu \mathrm{L}$ Lipofectamine 6000 were premixed in optiMEM to be added into the wells. After the cells were incubated $6 \mathrm{~h}$, opti-MEM was removed. Then FYGL at designated concentrations was added and incubated for another $24 \mathrm{~h}$. Finally, RNA and protein were extracted for qPCR and western blot analysis, respectively. Meanwhile ROS and ATP levels were also measured, respectively.

\subsection{Statistical analysis}

All data are presented as mean $\pm \mathrm{SD}$. Multi-group comparisons of the means were carried out by one-way analysis of variance (ANOVA) test with post hoc contrasts by Student-NewmanKeuls test. A difference is considered statistically to be significant when the $P$ value $<0.05$.

\section{Results}

\subsection{FYGL increases mtDNA copy number in skeletal muscle tissues of mice}

Fig. 1 shows the relative mtDNA copy number in skeletal muscle tissues of mice treated with saline, FYGL and metformin, respectively. The mtDNA level in skeletal muscle tissues of ob/ ob mice was remarkably lower than that of WT mice. However, FYGL orally administrated significantly up-regulated mtDNA content, similar to metformin. Interestingly, it was almost 1.5 times of mtDNA copy number in ob/ob mice treated with $400 \mathrm{mg} \mathrm{kg}^{-1}$ FYGL as much as that in WT group, demonstrating that FYGL can promote mitochondrial biogenesis. Therefore, it is interesting for us to investigate the related pathway which is possibly influenced by $F Y G L$.

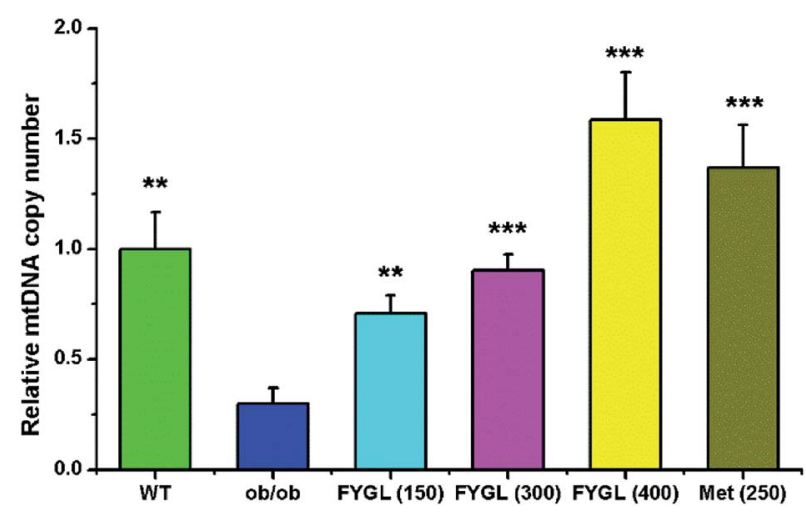

Fig. 1 The relative mtDNA copy number in skeletal muscle tissues of mice. The mtDNA copy number in WT mice is normalized to 1.0. Data are presented as mean $\pm \mathrm{SD}(n=6)$. $* * P<0.01, * * * P<0.001$ versus with ob/ob group.

\subsection{FYGL regulates mitochondrial signaling pathway}

Fig. 2 shows the relative expressions of mRNAs related to mitochondrial signaling pathway in skeletal muscle tissues of mice. All the expressions of mRNAs including Sirt1, Sirt3, PGC$1 \alpha$, NRF1 and TFAM were notably decreased in ob/ob mice, compared with that in WT group. However, the expressions of mRNAs were increased in ob/ob mice after treated with $150 \mathrm{mg}$ $\mathrm{kg}^{-1} F Y G L$, and significantly up-regulated when dose of $F Y G L$ was higher than $300 \mathrm{mg} \mathrm{kg}^{-1}$.

From the analysis data of western blot in Fig. 3, the proteins related to mitochondrial biogenesis including Sirt1, Sirt3, PGC$1 \alpha$, NRF1 and TFAM were all down-regulated in ob/ob mice, compared with that in WT group, and both the expressions of Sirt1 and Sirt3 were markedly up-regulated in ob/ob mice treated with $150 \mathrm{mg} \mathrm{kg}^{-1}$ FYGL. Interestingly, all the proteins depicted in Fig. $3 \mathrm{~b}$ exhibited significant increase when ob/ob mice took FYGL more than $300 \mathrm{mg} \mathrm{kg}^{-1}$. The results of western blot were consistent with those of qPCR.

\subsection{FYGL decreases ROS level and increases ATP content in L6 cells}

For clearly understanding the effect of $F Y G L$ on mitochondrial biogenesis, we investigated ROS level and ATP content in L6 cells. From Fig. 4a, fluorescent intensity of DCF represents the relative ROS level, and that of control group is normalized to 1.0. FYGL significantly decreased ROS level in L6 cells when its concentration was higher than $100 \mu \mathrm{g} \mathrm{mL}^{-1}$. Furthermore, from Fig. 4b, ATP content was increased in L6 cells after incubated with FYGL. Interestingly, ATP level was significantly increased when concentration of $F Y G L$ was higher than $100 \mu \mathrm{g} \mathrm{mL} \mathrm{m}^{-1}$, which was similar to the result of ROS level in L6 cells incubated with $F Y G L$.

In Fig. 5a, it was almost two times of ROS level in PGC-1 $\alpha$ silent cells as high as that in normal cells, but decreased mildly after incubated with relative low concentration of FYGL. When FYGL concentration was higher than $100 \mu \mathrm{g}$ $\mathrm{mL}^{-1}$, ROS level was significantly ameliorated. From Fig. 5b, ATP content was markedly decreased in PGC-1 $\alpha$ silent cells compared with normal group, reflecting that the mitochondrial biogenesis was weakened when the expression of PGC$1 \alpha$ was declined. After the PGC-1 $\alpha$ silent cells were incubated with FYGL especially when the concentration was higher than $50 \mu \mathrm{g} \mathrm{mL} \mathrm{L}^{-1}$, intracellular ATP content was significantly enhanced.

\subsection{FYGL increases PGC-1 $\alpha$ in L6 cells at the mRNA and protein levels}

Table 3 shows the relative expressions of mRNAs and mtDNA in L6 cells transfected with PGC- $1 \alpha$ siRNA and incubated with FYGL at different concentrations. After L6 cells were transfected with PGC-1 $\alpha$ siRNA, the mRNA expression of PGC-1 $\alpha$ was decreased instantly, accompanied with Sirt1, Sirt3, NRF1, TFAM mRNA and mtDNA copy number. Incubation with $F Y G L$ gradually enhanced PGC- $1 \alpha$ mRNA expression in PGC- $1 \alpha$ silent cells, and increased Sirt1 and Sirt3 mRNA expressions 


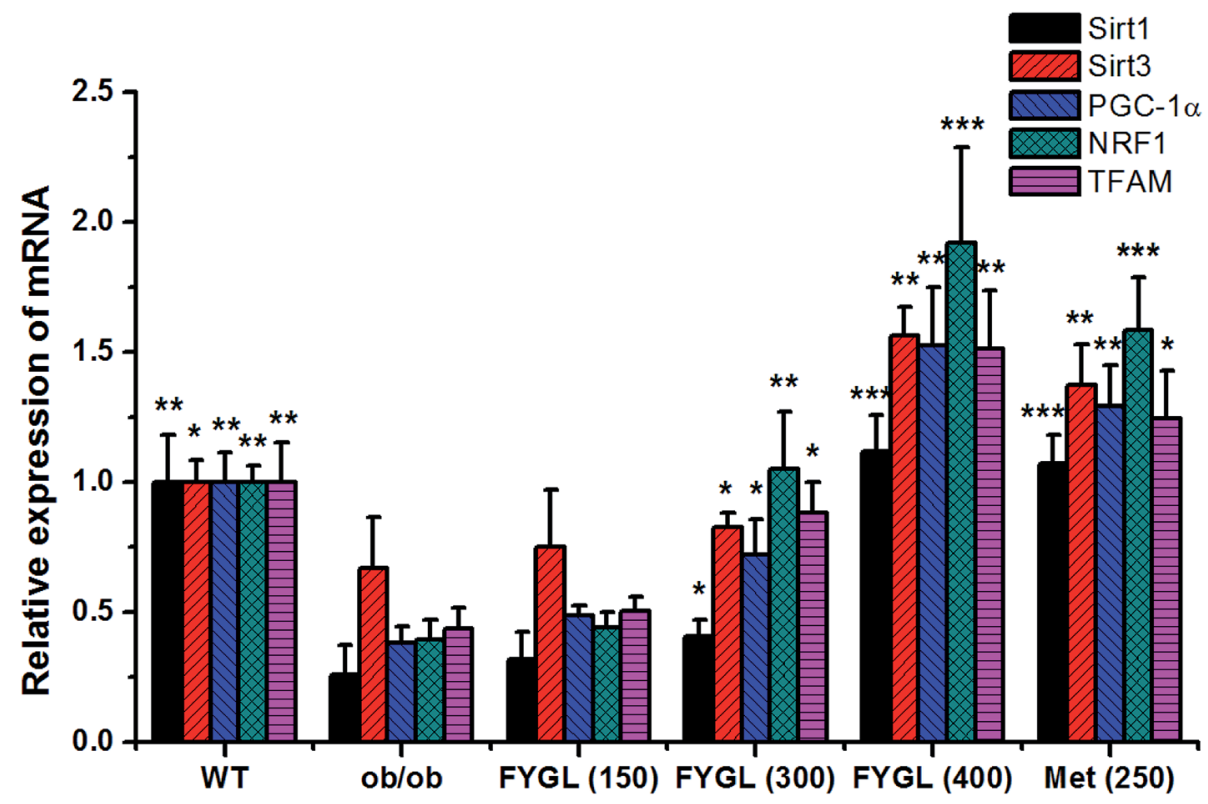

Fig. 2 The relative expression of mRNA including Sirt1, Sirt3, PGC-1 $\alpha$, NRF1 and TFAM in skeletal muscle tissues of mice. The expressions of mRNA in WT mice are normalized to 1.0. Data are presented as mean $\pm \mathrm{SD}(n=6)$. $* P<0.05, * * P<0.01, * * * P<0.001$ versus with ob/ob group.

when the concentration of $F Y G L$ was higher than $50 \mu \mathrm{g} \mathrm{mL} \mathrm{L}^{-1}$, along with effectively improved NRF1 and TFAM mRNA expressions. In accordance with TFAM mRNA, mtDNA copy number in L6 cells was also significantly increased after incubated with $F Y G L$.

As shown in Fig. 6, the expression of PGC- $1 \alpha$ was significantly decreased in PGC- $1 \alpha$ silent cells, followed with reductions of Sirt1, Sirt3, NRF1 and TFAM. Compared with control group, treatment with FYGL markedly increased PGC-1 $\alpha$, Sirt1, Sirt3, NRF1 and TFAM, especially when the concentration of FYGL was higher than $50 \mu \mathrm{g} \mathrm{mL}{ }^{-1}$, coinciding with the results of qPCR.

\section{Discussion and conclusions}

4.1 The mechanism of FYGL decreasing ROS and increasing ATP

In recent decades, ROS has been identified to be a major factor in the development of T2DM by inactivating signaling pathway from insulin receptor to glucose transporter, and overproduction of ROS may induce apoptosis of islet $\beta$ cells. ${ }^{7}$ FYGL was demonstrated to be a neutral hyperbranched proteoglycan consisting of 4 saccharides and 17 amino acids,${ }^{17}$ the reductive sugars of FYGL including glucose and galactose might play an important role in ameliorating ROS level. ${ }^{5}$ Our previous work
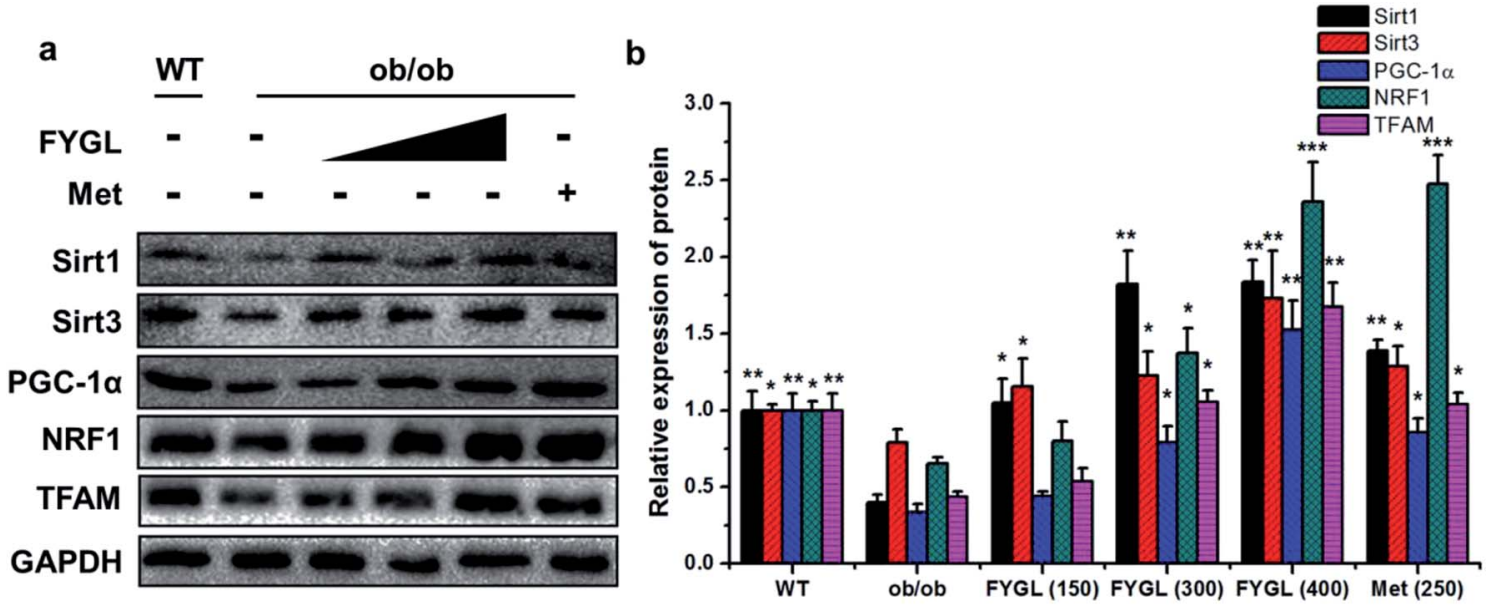

Fig. 3 Western blot analysis of proteins in mitochondrial signaling pathway in skeletal muscle tissues of mice. (a) Band images of relevant proteins: Sirt1, Sirt3, PGC-1 $\alpha$, NRF1, TFAM with GAPDH used as internal reference. (b) The relative expression of Sirt1, Sirt3, PGC-1 $\alpha$, NRF1 and TFAM. The expressions in WT mice are normalized to 1.0. Data are presented as mean \pm SD $(n=6)$. * $P<0.05, * * P<0.01, * * * P<0.001$ versus with ob/ob group. 
a

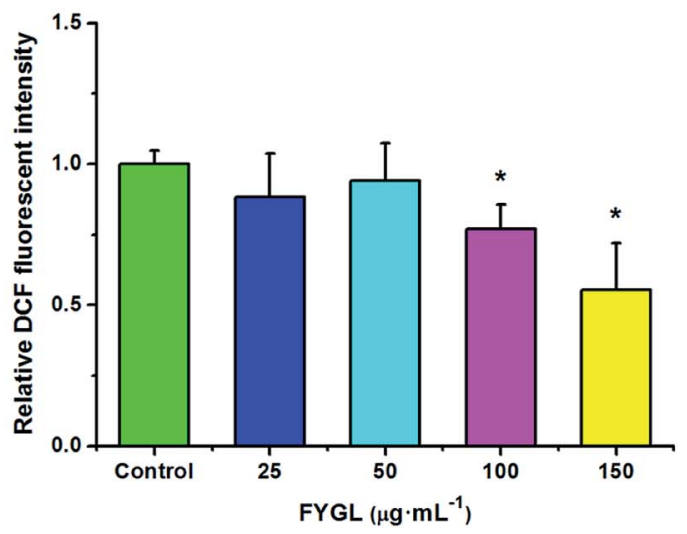

b

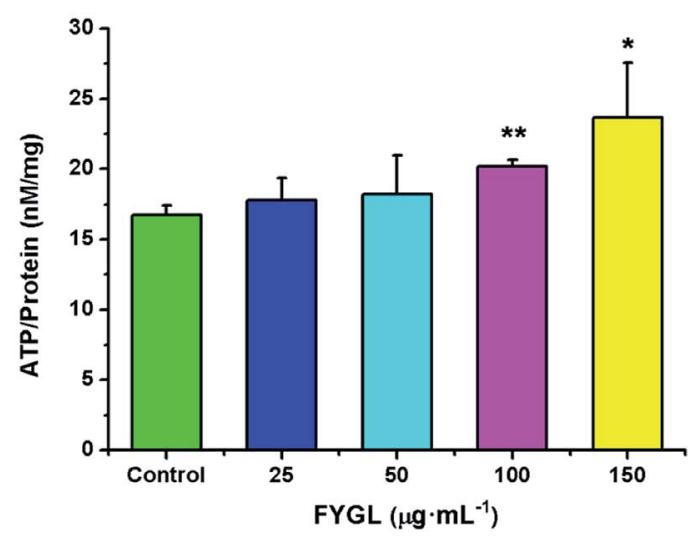

Fig. 4 (a) Relative DCF fluorescent intensity in L6 cells incubated with different concentrations of FYGL. The relative DCF fluorescent intensity in control group is normalized to 1.0. (b) ATP content in L6 cells incubated with different concentrations of FYGL. Data are presented as mean \pm SD $(n=3), * P<0.05$ versus with control group.

also found that FYGL can protect rat insulinoma cells (an islet $\beta$ cell mode) against impairment from the fibrillation of human islet amyloid polypeptide (hIAPP), ${ }^{18}$ which suggested FYGL protected islet $\beta$ cells possibly through ameliorating intracellular overproduction of ROS induced by misfolding of hIAPP. ${ }^{19}$

Overexpression of PGC- $1 \alpha$ can enhance intracellular ATP content, while deficiency of PGC- $1 \alpha$ often causes decrease of ATP in cardiomyocytes via adiponectin/AMPK/PGC-1 $\alpha$ pathway. ${ }^{20}$ Liu et al. ${ }^{21}$ reported that lead acetate can also increase ROS and decrease ATP in TM4 cells, accompanying with down-regulation of PGC- $1 \alpha$ and Sirt3 mRNA. In our previous work, FYGL was demonstrated to enhance adiponectin and promote phosphorylation of AMPK $\alpha$ in vivo. ${ }^{22}$ In the current study, FYGL can increase ATP content in addition to decrease of ROS level in PGC- $1 \alpha$ deficient cells, regulating mitochondrial biogenesis through PGC- $1 \alpha$ related pathway.

\subsection{Mitochondrial biogenesis regulated by FYGL}

Mitochondrial dysfunction is a complication of high serum lipid-induced ROS in skeletal muscle tissues, in turn promotes ROS and mitochondrial alteration. ${ }^{23}$ High serum lipid induces the reduction of mitochondrial density in insulin resistant skeletal muscle. ${ }^{24}$ In addition, mitochondrial dysfunction can also exacerbate T2DM by inhibiting insulin action, ${ }^{23}$ and the toxic by-product from ROS will induce cell apoptosis and inhibit signaling transduction. ${ }^{25}$ In our previous work, FYGL can downregulate serum lipid level, thus protecting peripheral tissues against damage from ROS. ${ }^{22}$ Moreover, mtDNA is also quite sensitive to ROS level and can be impaired by overload of ROS. ${ }^{25,26}$ Ikeda et al. reported that overexpression of TFAM can increase mtDNA copy number and limit mitochondrial oxidative stress. ${ }^{27}$ In this work the mtDNA copy number in skeletal muscle tissues of ob/ob mice was significantly lower than that of WT group, whereas treatment with FYGL remarkably up- a

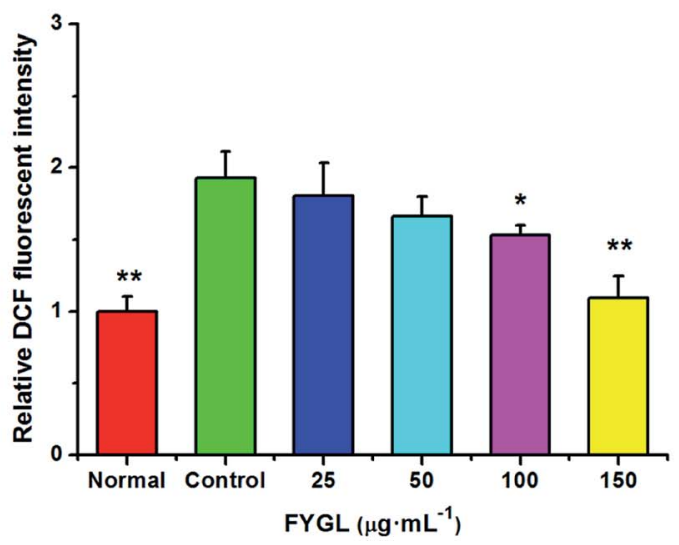

b

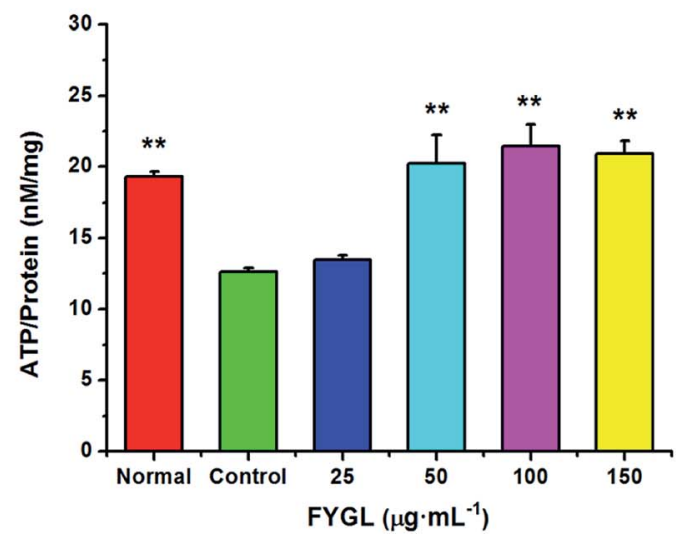

Fig. 5 (a) Relative DCF fluorescent intensity in PGC-1 $\alpha$ silent $L 6$ cells incubated with different concentrations of FYGL. The relative DCF fluorescent intensity in control group is normalized to 1.0. (b) ATP content in PGC-1 $\alpha$ silent $L 6$ cells incubated with different concentrations of FYGL. Data are presented as mean $\pm \mathrm{SD}(n=3), * P<0.05, * * P<0.01$ versus with control group. 
Table 3 The relative expressions of mRNA including Sirt1, Sirt3, PGC-1 $\alpha$, NRF1 and TFAM in L6 cells. The expressions of mRNA in normal L6 cells are normalized to $1.0^{a}$

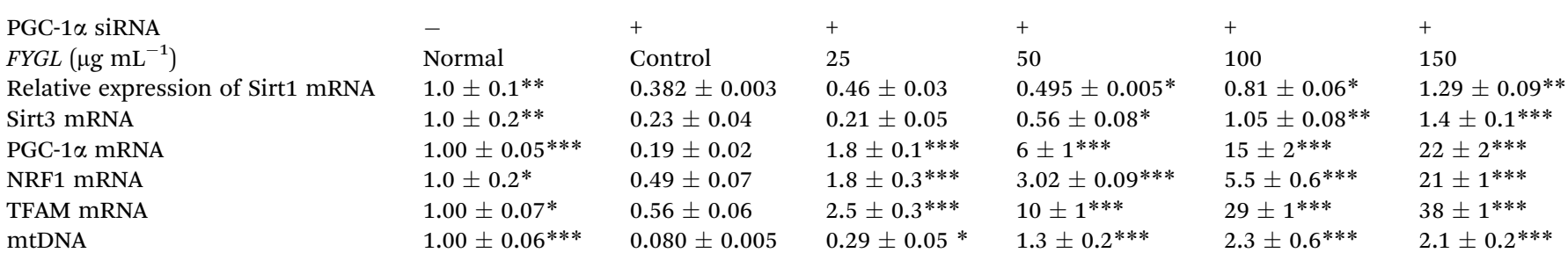

${ }^{a}$ Data are presented as mean $\pm \mathrm{SD}(n=3) .{ }^{*} P<0.05,{ }^{* *} P<0.01,{ }^{* * *} P<0.001$ versus with control group.

regulated mtDNA copy number and improved the expression of TFAM in ob/ob mice, indicating that FYGL can also improve the mitochondrial biogenesis.

\subsection{Influence of PGC-1 $\alpha$ by FYGL}

Overexpression of PGC- $1 \alpha$ can promote lipid metabolism and mitochondrial biogenesis ${ }^{3}$ and increase insulin sensitivity in skeletal muscle tissues. ${ }^{28}$ It was reported that aldosterone decreases mtDNA copy number and ATP content, while PGC$1 \alpha$ overexpression can protect mitochondria against aldosterone-induced podocyte depletion. ${ }^{11}$ Knockdown of PGC- $1 \alpha$ leads a series of mitochondrial alterations including aggravation of oxidative stress and fatty acid oxidation, ${ }^{29}$ even exacerbates insulin resistance. ${ }^{30}$ Wan et al. ${ }^{31}$ reported that PGC- $1 \alpha$ is regulated by activation of AMPK. In this work, the expression of PGC- $1 \alpha$ was significantly decreased in skeletal muscle tissues of ob/ob mice along with reduction of mtDNA copy number, while FYGL remarkably increased expression of PGC-1 $\alpha$ and activated it major upstream regulator, AMPK, in vivo and in vitro. ${ }^{15}$

\subsection{Influence of energy metabolism pathway by $F Y G L$}

Energy demand activates intracellular AMPK, and then increases the ratio of NAD+/NADH, finally activated Sirt1/PGC-
$1 \alpha$ cascades. $^{2}$ Sirt1/PGC- $1 \alpha$ is a crucial metabolic adaptation which can integrate muscle cells in response to low nutrient, and Sirt1 can promote the utilization of mitochondrial fatty acid and the transcription of related gene factors. ${ }^{32}$ Sirt1 overexpression can improve insulin sensitivity and ameliorate ROS and insulin resistance in high-fat diet rats. ${ }^{13}$ In addition, PGC$1 \alpha$ stimulates Sirt3, and Sirt3 in turn regulates PGC- $1 \alpha$ through phosphorylation of cAMP-response element binding protein (CREB). ${ }^{33}$ Knockdown of Sirt3 significantly suppresses PGC-1 $\alpha$ and expressions of other mitochondrial related genes, meanwhile decreases mitochondrial copy number and increases ROS level. ${ }^{33}$ PGC- $1 \alpha$ can bind and co-activate NRF1, and increase transcriptional activity on target gene including TFAM. ${ }^{34}$ Kang et $a .^{35}$ reported that aspartate raised ATP content in liver through AMPK-Sirt1-PGC-1 signal pathway. Intriguingly, FYGL promoted phosphorylation of AMPK in skeletal muscle tissues of ob/ob mice, ${ }^{15}$ and also increased ATP content in L6 cells through activating Sirt1/PGC-1 $\alpha$ cascades. Although the mitochondrial related gene expressions were all down-regulated in skeletal muscle tissues of ob/ob mice, FYGL orally administrated significantly up-regulated Sirt1/TFAM cascades at the mRNA level and protein level. STR1720, a Sirt1 specific activator reported by Feige et $a l .{ }^{36}$ can ameliorate insulin resistance in muscle, liver and brown adipose tissues, but its function is different from $F Y G L$. a

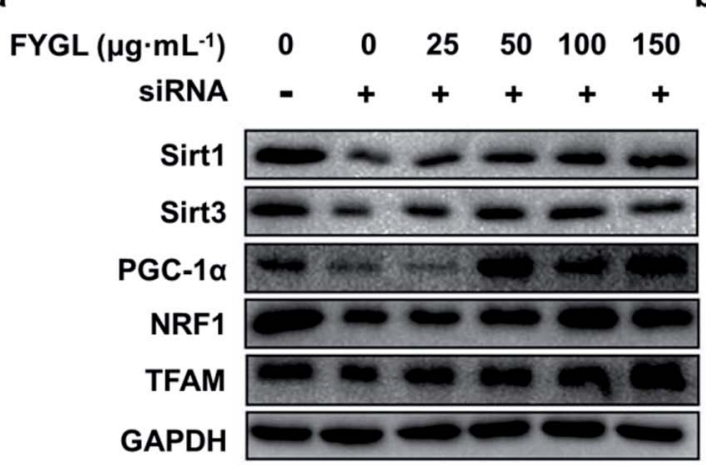

\section{b}

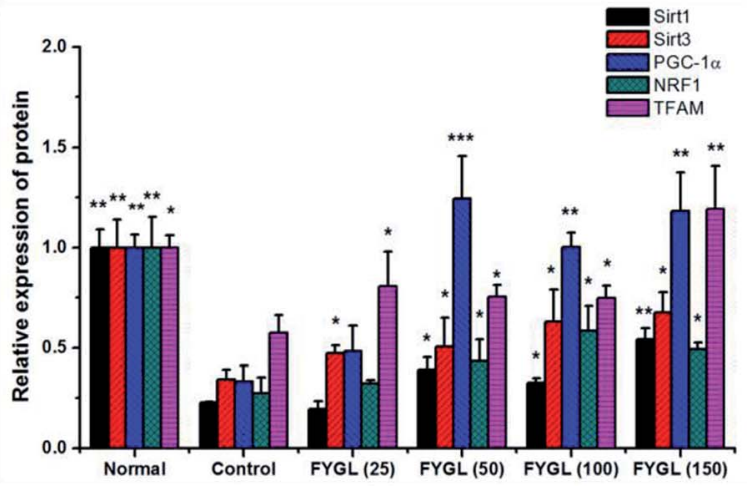

Fig. 6 Western blot analysis of proteins including Sirt1, Sirt3, PGC-1 $\alpha$, NRF1 and TFAM in L6 cells. (a) Band images of relevant proteins: Sirt1, Sirt3, PGC-1 $\alpha$, NRF1, TFAM with GAPDH used as internal reference. (b) The relative expressions of Sirt1, Sirt3, PGC-1 $\alpha$, NRF1 and TFAM. The expressions in normal L6 cells are normalized to 1.0. Data are presented as mean \pm SD $(n=3) . * P<0.05, * * P<0.01, * * * P<0.001$ versus with control group. 


\subsection{Conclusions}

In summary, our work investigated the effects of $F Y G L$ on mitochondrial biogenesis on the basis of the evidence that FYGL can regulate blood glucose and ameliorate insulin resistance in ob/ob mice. We found that FYGL ameliorated cellular damage from ROS and promoted ATP synthesis in L6 cells, along with enhancement of PGC- $1 \alpha$ expression. Moreover, FYGL can also increase mtDNA copy number and expressions of mitochondrial related genes in vivo, thus protecting mitochondria against impairment from insulin resistance, in turn ameliorating insulin resistance.

\section{Author contributions}

Zhou Yang and Juan Zhao performed the experiments and analyzed the data. Zhou Yang and Ping Zhou designed all the experiments. Zhou Yang, Hongjie Yang and Ping Zhou wrote the manuscript.

\section{Conflicts of interest}

The authors claim that the researchers in this study have no conflict of interest.

\section{Abbreviations}

\begin{tabular}{|c|c|}
\hline AMP & Adenosine $5^{\prime}$-monophosphate \\
\hline AMPK & $\begin{array}{l}\text { Adenosine } 5^{\prime} \text {-monophosphate activated protein } \\
\text { kinase }\end{array}$ \\
\hline ATP & Adenosine-triphosphate \\
\hline CREB & cAMP-response element binding protein \\
\hline $\begin{array}{l}\text { DCFH- } \\
\text { DA }\end{array}$ & $2^{\prime}, 7^{\prime}$-Dichlorodihydrofluorescein diacetate \\
\hline mtDNA & Mitochondrial DNA \\
\hline NRF1 & Nuclear respiratory factor-1 \\
\hline PGC- $1 \alpha$ & $\begin{array}{l}\text { Peroxisome proliferator-activated receptor- } \gamma \\
\text { coactivator- } 1 \alpha\end{array}$ \\
\hline ROS & Reactive oxygen species \\
\hline Sir2 & Silent information regulator 2 \\
\hline Sirt1 & Sirtuin 1 \\
\hline Sirt3 & Sirtuin 3 \\
\hline T2DM & Type 2 diabetes mellitus \\
\hline TFAM & Mitochondrial transcription factor A \\
\hline
\end{tabular}

\section{Acknowledgements}

This work was supported by the Natural Science Foundation of China (No. 21374022 and 81374032), the Scientific Program of Shanghai Municipal Public Health Bureau (No. 2008220 and 2010231), the Science and Technology Innovation Action Plan of STCSM (No. 11DZ1971802), National Major Scientific and Technological Special Project for "Significant New Drugs Development” (No. 2017ZX09301006), Shanghai Science and Technology Innovation Action Plan "Science and Technology Support Project in Biomedical Science” (No. 17401902700).

\section{References}

1 M. Picard, T. Taivassalo, G. Gouspillou and R. T. Hepple, J. Photosci., 2011, 589, 4413-4421.

2 D. C. Wallace, Science, 1999, 283, 1482-1488.

3 S. D. Martin and S. L. McGee, Biochim. Biophys. Acta, 2014, 1840, 1303-1312.

4 J. Szendroedi, E. Phielix and M. Roden, Nat. Rev. Endocrinol., 2011, 8, 92-103.

5 P. Newsholme, E. P. Haber, S. M. Hirabara, E. L. Rebelato, J. Procopio, D. Morgan, H. C. Oliveira-Emilio, A. R. Carpinelli and R. Curi, J. Photosci., 2007, 583, 9-24.

6 K. Morino, K. F. Petersen, S. Dufour, D. Befroy, J. Frattini, N. Shatzkes, S. Neschen, M. F. White, S. Bilz, S. Sono, M. Pypaert and G. I. Shulman, J. Clin. Invest., 2005, 115, 3587-3593.

7 D. E. Kelley, J. He, E. V. Menshikova and V. B. Ritov, Diabetes, 2002, 51, 2944-2950.

8 V. K. Mootha, C. M. Lindgren, K. F. Eriksson, A. Subramanian, S. Sihag, J. Lehar, P. Puigserver, E. Carlsson, M. Ridderstrale, E. Laurila, N. Houstis, M. J. Daly, N. Patterson, J. P. Mesirov, T. R. Golub, P. Tamayo, B. Spiegelman, E. S. Lander, J. N. Hirschhorn, D. Altshuler and L. C. Groop, Nat. Genet., 2003, 34, 267-273.

9 K. F. Petersen, D. Befroy, S. Dufour, J. Dziura, C. Ariyan, D. L. Rothman, L. DiPietro, G. W. Cline and G. I. Shulman, Science, 2003, 300, 1140-1142.

10 P. J. Fernandez-Marcos and J. Auwerx, Am. J. Clin. Nutr., 2011, 93, 884S-890S.

11 M. Zhao, Y. Yuan, M. Bai, G. Ding, Z. Jia, S. Huang and A. Zhang, Oncotarget, 2016, 7, 12150-12162.

12 B. L. Tang, Mol. Cells, 2016, 39, 87-95.

13 H. H. Zhang, G. J. Qin, X. L. Li, Y. H. Zhang, P. J. Du, P. Y. Zhang, Y. Y. Zhao and J. Wu, J. Physiol. Biochem., 2015, 71, 177-190.

14 B. S. Teng, C. D. Wang, H. J. Yang, J. S. Wu, D. Zhang, M. Zheng, Z. H. Fan, D. Pan and P. Zhou, J. Agric. Food Chem., 2011, 59, 6492-6500.

15 Z. Yang, F. Wu, Y. He, Q. Zhang, Y. Zhang, G. Zhou, H. Yang and P. Zhou, Food Funct., 2018, 9, 397-406.

16 H. A. Erlich, D. H. Gelfand and R. K. Saiki, Nature, 1988, 331, 461-462.

17 D. Pan, L. Wang, C. Chen, B. Hu and P. Zhou, Carbohydr. Polym., 2015, 117, 106-114.

18 Q. Sun, J. Zhao, Y. Zhang, H. Yang and P. Zhou, RSC Adv., 2016, 6, 105690-105698.

19 X. L. Li, Y. S. Wong, G. Xu and J. C. Chan, Eur. J. Nutr., 2015, 54, 509-522.

20 W. Yan, H. Zhang, P. Liu, H. Wang, J. Liu, C. Gao, Y. Liu, K. Lian, L. Yang, L. Sun, Y. Guo, L. Zhang, L. Dong, W. B. Lau, E. Gao, F. Gao, L. Xiong, H. Wang, Y. Qu and L. Tao, Basic Res. Cardiol., 2013, 108, 329.

21 X. Liu, J. Ye, L. Wang, Z. Li, Y. Zhang, J. Sun, C. Du, C. Wang and S. Xu, Biol. Trace Elem. Res., 2017, 175, 440-448.

22 Z. Yang, C. Chen, J. Zhao, W. Xu, Y. He, H. Yang and P. Zhou, Eur. J. Pharmacol., 2017, 820, 77-85. 
23 S. Di Meo, S. Iossa and P. Venditti, J. Endocrinol., 2017, 233, R15-R42.

24 P. Chomentowski, P. M. Coen, Z. Radikova, B. H. Goodpaster and F. G. Toledo, J. Clin. Endocrinol. Metab., 2011, 96, 494503.

25 K. Brieger, S. Schiavone, F. J. Miller Jr and K. H. Krause, Swiss Med. Wkly., 2012, 142, w13659.

26 T. Ide, H. Tsutsui, S. Hayashidani, D. C. Kang, N. Suematsu, K. Nakamura, H. Utsumi, N. Hamasaki and A. Takeshita, Circ. Res., 2001, 88, 529-535.

27 M. Ikeda, T. Ide, T. Fujino, S. Arai, K. Saku, T. Kakino, H. Tyynismaa, T. Yamasaki, K. Yamada, D. Kang, A. Suomalainen and K. Sunagawa, PLoS One, 2015, 10, e0119687.

28 C. R. Benton, J. G. Nickerson, J. Lally, X. X. Han, G. P. Holloway, J. F. Glatz, J. J. Luiken, T. E. Graham, J. J. Heikkila and A. Bonen, J. Biol. Chem., 2008, 283, 42284240.

29 M. Waldman, L. Bellner, L. Vanella, J. Schragenheim, K. Sodhi, S. P. Singh, D. Lin, A. Lakhkar, J. Li,
E. Hochhauser, M. Arad, Z. Darzynkiewicz, A. Kappas and N. G. Abraham, Stem Cells Dev., 2016, 25, 1084-1094.

30 S. Kleiner, R. J. Mepani, D. Laznik, L. Ye, M. J. Jurczak, F. R. Jornayvaz, J. L. Estall, D. Chatterjee Bhowmick, G. I. Shulman and B. M. Spiegelman, Proc. Natl. Acad. Sci. U. S. A., 2012, 109, 9635-9640.

31 Z. Wan, J. Root-Mccaig, L. Castellani, B. E. Kemp, G. R. Steinberg and D. C. Wright, Obesity, 2014, 22, 730-738. 32 Z. Gerhart-Hines, J. T. Rodgers, O. Bare, C. Kim, S. H. Kim, R. Mostoslavsky, F. W. Alt, Z. D. Wu and P. Puigserver, EMBO J., 2007, 26, 1913-1923.

33 X. Kong, R. Wang, Y. Xue, X. Liu, H. Zhang, Y. Chen, F. Fang and Y. Chang, PLoS One, 2010, 5, e11707.

34 Z. Wu, P. Puigserver, U. Andersson, C. Zhang, G. Adelmant, V. Mootha, A. Troy, S. Cinti, B. Lowell, R. C. Scarpulla and B. M. Spiegelman, Cell, 1999, 98, 115-124.

35 P. Kang, Y. Liu, H. Zhu, S. Li, H. Shi, F. Chen, W. Leng, D. Pi, Y. Hou and D. Yi, Eur. J. Nutr., 2015, 54, 581-588.

36 J. N. Feige, M. Lagouge, C. Canto, A. Strehle, S. M. Houten, J. C. Milne, P. D. Lambert, C. Mataki, P. J. Elliott and J. Auwerx, Cell Metab., 2008, 8, 347-358. 